\title{
Coisotropic regularization of singular Lagrangians
}

\author{
A. Ibort \\ Departamento de Física Teórica, Universidad Complutense de Madrid, \\ 28040 Madrid, Spain
}

\author{
J. Marín-Solano \\ Departamento de Matematica Economica, Financera i Actuarial, \\ Universitat de Barcelona, 08034 Barcelona, Spain
}

(Received 31 October 1994; accepted for publication 5 July 1995)

We present an alternative approach to the usual treatments of singular Lagrangians. It is based on a Hamiltonian regularization scheme inspired on the coisotropic embedding of presymplectic systems. A Lagrangian regularization of a singular Lagrangian is a regular Lagrangian defined on an extended velocity phase space that reproduces the original theory when restricted to the initial configuration space. A Lagrangian regularization does not always exists, but a family of singular Lagrangians is studied for which such a regularization can be described explicitly. These regularizations turn out to be essentially unique and provide an alternative setting to quantize the corresponding physical systems. These ideas can be applied both in classical mechanics and field theories. Several examples are discussed in detail. (C) 1995 American Institute of Physics.

\section{INTRODUCTION}

Singular Lagrangians are commonplace in physical theories. In most cases the degeneracy of the Lagrangian function is directly related to the gauge invariance of the theory. This happens, for instance, in gauge theories such as Yang-Mills theories, gravitation, etc. ${ }^{1}$ In other occasions, the Lagrangian of the theory becomes singular in a particular sector of the physical model under consideration, for instance, the Chern-Simons Lagrangian arises as the zero mass limit of a massive Yang-Mills theory in $(2+1)$ dimensions with a topological term. ${ }^{2}$ A similar finitedimensional problem will arise when we consider, for instance, the system

$$
L=\frac{1}{2} m_{i}\left(\dot{q}^{i}\right)^{2}+e A_{i}(q) \dot{q}^{i}-V(q)
$$

in the limit $m_{i} \rightarrow 0$ for some $i$. Finally, there are some situations where the Lagrangian of a physical system is ill defined (i.e., it is defined only locally) and a remedy to this "disease" is to extend the configuration space of the system in such a way that there is a well-defined Lagrangian on it. ${ }^{3,4}$ This is the case, for instance, for the electron-monopole system. It usually results that the global Lagrangian in the extended configuration space is singular.

All these situations can be dealt with at the classical level either using ad hoc methods specific to the examples at hand, or in more a systematic way, using Dirac's theory of constraints. ${ }^{5}$ At the quantum level, a powerful tool has emerged in the last twenty years, the BRST-BFV quantization scheme (see, for instance, Ref. 6 and references therein), providing a covariant quantization scheme for constrained Hamiltonian systems.

In this article we propose an alternative procedure to deal with singular Lagrangians at the classical level: instead of using the Hamiltonian formalism and Dirac's theory of constraints, we remain in the velocity phase space of the system, i.e., in the Lagrangian setting. Then we will try to find an extension of such space possessing a regular Lagrangian leading to an equivalent theory once the extra degrees of freedom that have been introduced are removed. We will call such a system a Lagrangian regularization of the original singular Lagrangian. Had we not required that the regularized theory be equivalent to the initial one restricted to the original velocity phase 
space, then we could have simply regularized an arbitrary Lagrangian adding a kinetic energy term. For instance, in the finite-dimensional example above, Eq. (1), a term of the form $\frac{1}{2} \epsilon q_{i}^{2}$ will regularize the Lagrangian with $m_{i}=0$. In doing that, we are changing the dynamical content of the theory and spoiling the gauge invariance derived from the singular character of the Lagrangian. Thus, a regularization scheme for singular Lagrangians must include the prescription to generate the gauge symmetry that the singular Lagrangian defines as well as the constraints in the phase space of the system.

It is evident that once a Lagrangian regularization has been found, we can quantize the regular system in the extended velocity phase space and, later on, eliminate the spurious degrees of freedom introduced in the process. It is also evident that we can imagine ad hoc methods to quantize our singular Lagrangian. For instance, considering again the finite-dimensional example above, Eq. (1), a possible way to proceed simply consists of putting the mass $m_{i} \neq 0$ back, quantizing, and afterwards, again taking the limit $m_{i} \rightarrow 0$. However such a limit is ambiguous because we do not know which constraints we must put on the physical states of the theory. In the general case, contrary to what happens in this example, there is not even an obvious way to proceed, so the systematic procedure offered hy a I agrangian regularization can be of interest. Another advantage of a Lagrangian regularization scheme is that it preserves the symmetries of the Lagrangian in an obvious way and we do not have to pass to the Hamiltonian formalism.

If a Lagrangian regularization exists for the singular Lagrangian $L$, in principle it is not unique and there could be many different ways of regularizing a given singular Lagrangian leading to different quantum theories. However, we will show, rather surprisingly, that for an interesting class of singular Lagrangians, if such regularization exists, it is essentially unique. Such Lagrangians are contained in the family of type II Lagrangians described in Ref. 7.

The regularization scheme for singular Lagrangians that we are proposing is based on a (bosonic) extension of the velocity phase space by first class constraints introduced by Gotay and Sniatycki ${ }^{8}$ to geometrically quantize general presymplectic systems. We will call it a Hamiltonian regularization of the singular Lagrangian under consideration. The first class constraints defining the original velocity phase space inside the extended phase space can be thought of as defining a generalized (Abelian) symmetry that allows us to recover Dirac's formalism. The example in Sec. II $D$ shows how to implement the Hamiltonian regularization scheme for a Lagrangian system linear in velocities. We will prove first the equivalence of the Hamiltonian regularization with Dirac's formalism (in the Lagrangian setting) for systems without secondary constraints. Later on the general case is considered and the equivalence with Dirac's formalism in the Lagrangian setting is proven.

A regularization of the singular Lagrangian $L$ allows us to pass to the Hamiltonian formalism using the corresponding Legendre transformation. We will show that the constraints introduced in the Lagrangian setting can be used in the Hamiltonian formalism in the corresponding extended phase space to generate an extended Dirac's theory. In the presence of higher order constraints both theories could give different answers as it is shown, for instance, by the example in Sec. IV A. This is a common feature of singular Lagrangians without any regularization scheme (however for semiregular Lagrangian, both approaches give the same theory). This example also shows that Dirac's theory of constraints can lead to a void or uninteresting systems whereas, the proposed regularization schemes give reasonable answers (already at the classical level).

The construction of a Lagrangian regularization uses extensively the geometry of tangent bundles. These geometrical requirements isolate the family of type II Lagrangians as natural candidates for possessing such a regularization. The construction of a Lagrangian regularization for the singular Lagrangian $L_{0}$, i.e., the construction of a tangent structure in the phase space of its Hamiltonian regularization and the identification of the extended symplectic structure with the Cartan two-form of a regular Lagrangian function can be shown explicitly if a technical condition is satisfied (Sec. III B) (the characteristic distribution being of tangent type). Such a condition, even if sufficient is not necessary as is shown in examples. Then, the inverse problem of the 
calculus of variations is used to construct explicitly the regularized Lagrangian for the given system.

The article will be organized as follows. In Sec. II, basic facts of the geometry of Lagrangian systems and the Hamiltonian regularization of presymplectic systems will be discussed. Section III will be devoted to discuss the existence of a regularized Lagrangian for an interesting class of Lagrangians including many physical situations. The technical discussion will be carried out in finite dimensions and the geometrical ideas involved in the construction will be emphasized, even though the discussion can be carried out in the infinite-dimensional case without too much trouble. It will be illustrated with the discussion of the Lagrangian regularization of the electromagnetic Lagrangian in Sec. IV B.

\section{SINGULAR LAGRANGIANS AND HAMILTONIAN REGULARIZATION OF PRESYMPLECTIC SYSTEMS}

Let $L_{0}$ be a Lagrangian defined on the velocity phase space $T Q$ of a configuration space $Q$, that will be supposed to be a smooth manifold with local coordinates $q^{i}$. There is a canonical (1,1)-tensor field $S$ on $T Q$, called the vertical endomorphism, which is given by $S=\partial / \partial \dot{q}^{i} \otimes d q^{i}$ in local natural coordinates $\left(q^{i}, \dot{q}^{i}\right)$. The Cartan one-form associated to $L_{0}$ is defined by $\theta_{L_{0}}=d L_{0}$ - $S=\left(\partial L / \partial \dot{q}^{i}\right) d q^{i}$, and the Cartan two-form is given by $\omega_{L_{0}}=-d \theta_{L_{0}}$. The Lagrangian $L_{0}$ is called singular (nonregular or degenerate) if the Cartan two-form $\omega_{L_{0}}$ is degenerate. We will assume that the kernel $K$ of the Cartan two-form $\omega_{L_{0}}$ defines a smooth distribution on $T Q$, i.e., $K$ is a sub-bundle of $T(T Q)$. Under such circumstances $K$ defines an integrable distribution because $\omega_{L_{0}}$ is closed. The sections of $K$ will define a (possibly infinite-dimensional) Lie algebra that will be called the (primary) gauge algebra of the theory. Technically, the bundle $K \rightarrow T Q$ defines a Lie algebroid with anchor map, the natural identification $K \hookrightarrow T(T Q)$ of $K$ as a sub-bundle of $T(T Q)^{9}{ }^{9}$

The dynamics of the system defined by $L_{0}$ is given by a vector field $\Gamma_{0}$ satisfying the dynamical equation

$$
i_{\Gamma_{0}} \omega_{L_{0}}=d E_{L_{0}}
$$

where $E_{L_{0}}=\Delta\left(L_{0}\right)-L_{0}$ denotes the energy of the system, and $\Delta=v^{i} \partial / \partial v^{i}$ is the vertical vector field on $T Q$ generating dilations along the fibers. The existence of a vector field $\Gamma_{0}$ satisfying the dynamical equation (2) in all $T Q$, is equivalent to the nonexistence of dynamical secondary constraints. ${ }^{10.11}$ In this case the true physical phase space of the theory is the quotient space $R=T Q / K$. It will be supposed that $R$ is a smooth manifold and the canonical projection, denoted by $p: T Q \rightarrow R$, is a submersion. In general, a vector field satisfying Eq. (2) cannot be found in all points of $T Q$, thus a family of submanifolds $M_{k}$ can be defined recursively as follows:

$$
M_{0}=T Q ; \quad M_{k+1}=\left\{m \in M_{k} \mid \exists U \in T_{m} M_{k}, i_{U} \omega_{L_{0}}(m)=d E_{L_{0}}(m)\right\}, \quad k \in \mathbf{N} .
$$

If the sequence $M_{k}$ stabilizes, there is a well-defined (but not necessarily unique) dynamics on the final constraint submanifold $M_{\infty}=\cap_{k \geqslant 0} M_{k}$. Similarly the restriction of $\omega_{L_{0}}$ and $E_{L_{0}}$ to $M_{\infty}$ will be denoted by $\omega_{\infty}$ and $E_{\infty}$, respectively. We will assume that $\omega_{\infty}$ is a presymplectic form. The characteristic distribution $K_{\infty}=\operatorname{ker} \omega_{\infty}$, will be called the gauge algebra of the theory and the phase space of the theory will be now the reduced space $R=M_{\infty} / K_{\infty}$. Again it will be assumed that the canonical projection $p_{\infty}: M_{\infty} \rightarrow R$ is a smooth submersion. This construction will be referred to, in what follows, as the presymplectic constraint algorithm (PCA). ${ }^{10}$ In any case the reduced space $R$ inherits a symplectic structure $\omega_{R}$ because the presymplectic two-form $\omega_{\infty}$ is projectable along the fibration defined by $K_{\infty}$, and $\omega_{R}$ is related to $\omega_{\infty}$ by $p_{\infty}^{*} \omega_{R}=\omega_{\infty}$. The dynamical vector fields 
$\Gamma_{\infty}$ solutions of Eq. (2) in $M_{\infty}$, are also projectable along $K_{\infty}$ and define a vector field $\Gamma_{R}$ in $R$ which is Hamiltonian with Hamiltonian function $E_{R}$ defined by projecting $E_{\infty}$ (which is constant along the fibers of $p_{\infty}$ ).

Then, if the reduced phase space of the theory described by the singular Lagrangian $L_{0}$ is the Hamiltonian system $\left(R, \omega_{R}, \Gamma_{R}\right)$, the main problem now is to describe it both quantum and classically.

\section{A. Hamiltonian regularization of presymplectic systems}

There is an alternative way of obtaining the reduced Hamiltonian system $\left(R, \omega_{R}, E_{R}\right)$. We will use a regularization of the Lagrangian presymplectic system $\left(T Q, \omega_{L_{0}}, E_{L_{0}}\right)$ and we will apply to it a generalized symplectic reduction procedure. Such regularization can be explicitly constructed using the coisotropic embedding theorem as indicated in the Introduction. We will review succinctly the construction of a Hamiltonian regularization for general presymplectic systems ${ }^{8}$ and we will describe the generalized symmetry of this regularization.

Let $(M, \omega)$ be a presymplectic manifold. Given a function $H$, it defines locally Hamiltonian dynamical systems $\Gamma$ as vector fields satisfying the equation $i_{\Gamma} \omega=d H$. We will assume first that there exists a globally defined dynamical vector field $\Gamma$. If this were not the case, the presymplectic constraint algorithm discussed in the previous section should be used to obtain the final constraint submanifold defining the adequate presymplectic system $\left(M_{\infty}, \omega_{\infty}, H_{\infty}\right)$ suitable for the discussion to follow (see Sec. II $E$ for further discussion on this). Denoting again by $K$ the characteristic distribution of $\omega$, the true physical space of the theory is the quotient space $R=M / K$. The dynamics $\Gamma$ descends to the quotient $R$ defining an induced dynamics $\Gamma_{R}$ and $H$ is constant along the fibers of $p$.

The presymplectic system defined by $(M, \omega, H)$ can be regularized, that is, there exists a regular Hamiltonian system on which the presymplectic system is embedded and from which the reduced phase space can be obtained via generalized symplectic reduction. The coisotropic embedding theorem states for a presymplectic manifold $(M, \omega)$ the existence of a symplectic manifold $(P, \Omega)$ and an embedding $j: M \rightarrow P$ such that $j^{*} \Omega=\omega$ and $M$ is a coisotropic submanifold (that is, a first class submanifold) of $P$. The symplectic manifold $P$ is constructed as a tubular neighborhood of the zero section of the vector bundle $\pi: K^{*} \rightarrow M$, and $\Omega$ is constructed using a splitting of the tangent space of $K^{*}$ along $M$, i.e.,

$$
T_{M} K^{*} \cong K^{*} \oplus T M \cong K^{*} \oplus K \oplus F,
$$

where $F$ is a symplectic bundle that we can identify, for instance, with $K^{\perp}$ using a Riemannian metric on $M$. Then, the symplectic two-form $\Omega$ is defined as an extension of the two-form defined on $T_{M} K^{*}$ by $\omega+\omega_{K}$ to a tubular neighborhood of $M$ in $K^{*}$ using Weinstein's extension theorem. Here $\omega_{K}$ denotes the linear two-form canonically defined in the space $K \oplus K^{*}$ (see Ref. 12 for a detailed description).

It is important to remark here that all coisotropic embeddings of $(M, \omega)$ are symplectically equivalent in a neighborhood of $M$. That is, if $j_{a}: M \rightarrow\left(P_{a}, \Omega_{a}\right), a=1,2$, are two coisotropic embeddings, then there are two neighborhoods $U_{a}$ of $j_{a}(M)$ in $P_{a}$, and a symplectomorphism $\psi:\left(U_{1}, \Omega_{1}\right) \rightarrow\left(U_{2}, \Omega_{2}\right)$ such that $\psi \circ j_{1}=j_{2}$.

The construction is completed choosing a function $H_{P}$ such that $j^{*} H_{P}=H$. For instance, this can be done in a tubular neighborhood of $M$ in $K^{*}$ by pulling $H$ back using the canonical projection map $\pi: K^{*} \rightarrow M$, then $H_{P}=\pi^{*} H$. We have defined in this way a locally Hamiltonian dynamical system $\left(P, \Omega, H_{P}\right)$ with the desired properties.

\section{B. Coisotropic Hamiltonian regularization and generalized symplectic reduction}

Let $\left(P, \Omega, H_{P}\right)$ be a Hamiltonian regularization of the presymplectic system $(M, \omega, H)$. If we denote by $J$ the canonical embedding of $P$ in $K^{*}$, we can consider this map as a generalized 
momentum mapping $J: P \rightarrow K^{*}$. Notice that $\pi^{\circ} J \circ j=\mathrm{id}_{M}$ and then the zero level set of this generalized momentum mapping is precisely $M$, that is, $M=J^{-1}(Z)$, where $Z$ is the zero section of the vector bundle $\pi: K^{*} \rightarrow M$.

Associated to any section $\xi$ of $K$, there is a function $f_{\xi}$ on $K^{*}$ defined by $f_{\xi}(\mu)=\langle\mu, \xi(\pi(\mu))\rangle$, $\mu \in K^{*}$. The manifold $K^{*}$ carries a natural Poisson structure induced by the Lie algebroid $K{ }^{9}$ The Poisson bracket $\{.,\}_{K}$ on $K^{*}$ is given by

$$
\left\{f_{\xi}, f_{\zeta}\right\}_{K}=f_{[\xi, \zeta]} ; \quad\left\{f_{\xi}, f \circ \pi\right\}_{K}=\xi(f) \circ \pi ; \quad\{f \circ \pi, g \circ \pi\}_{K}=0
$$

for every $f, g \in C^{\infty}(M)$, and any sections $\xi, \zeta$ in $K$. Similarly, associated to any $\xi \in \Gamma(K)$, there is a function $J_{\xi}$ on $P$ defined by $J_{\xi}(x)=\langle J(x), \xi(\pi(J(x)))\rangle$. Notice that $f_{\xi^{\circ}} J=J_{\xi}$. The map $\check{J}: \Gamma(K) \rightarrow C^{\infty}(P)$ defined by $\breve{J}(\xi)=J_{\xi}$, is a generalized comomentum map that satisfies

$$
\left\{J_{\xi}, J_{\zeta}\right\}_{P}=J_{[\xi, \zeta]}, \quad \forall \xi, \zeta \in \Gamma(K),
$$

where $\{.,\}_{P}$ is the Poisson bracket defined by $\Omega$ in $P$. The previous identity (5) shows that $\check{J}$ is a homomorphism of Lie algebras. From Eqs. (4) and (5) we conclude that the generalized momentum map $J$ is a Poisson map when restricted to linear functions on $K^{*}$. We have completed in this way the parallelism with ordinary symplectic reduction. In fact, it can also be proven that if the Lie algebra $\Gamma(K)$ is finite dimensional, i.e., there exists a basis of vector fields $\xi_{i}$ such that $\left[\xi_{i}, \xi_{j}\right]=c_{i j}^{k} \xi_{k}$, then the vector bundles $K \rightarrow M$ and $K^{*} \rightarrow M$ are trivial. In particular $K^{*} \cong M \times V$ where $V$ is a vector space dual to the Lie algebra $\Gamma(K)$. Then $p r_{2}{ }^{\circ} J$ becomes the ordinary momentum map defined by restricting the action of the Lie algebra $\Gamma(K)$ on $P$.

We can apply now the usual ideas in reduction theory to the generalized momentum map $J$, i.e., we define the reduced phase space as the quotient of $J^{-1}(Z)$ by the characteristic distribution of the degenerate form defined by the restriction of $\Omega$ to $J^{-1}(Z)$. Then, it is obvious that

$$
J^{-1}(Z) /\left(\left.\operatorname{ker} \Omega\right|_{J^{-1}(Z)}\right)=M / K
$$

that is, the space obtained in this way is exactly the reduced phase space $R$.

The dynamics $H_{P}$ on $(P, \Omega)$ can be constructed adding any first class function to the pullback to $P$ of the energy function $H$ on $M$. For instance, if we choose a metric $\eta$ on the fiber bundle $K \rightarrow M$ we can define a Hamiltonian function by

$$
H_{P}(z, b)=\frac{1}{2} \eta^{\mu \nu}(z) b_{\mu} b_{\nu}+H(z)
$$

where $b_{\mu}$ are linear coordinates along the fibers of $K^{*} \rightarrow M$, and $z$ is a collective coordinate on $M$. In the particular case we are regularizing a Lagrangian system $\left(T Q, \omega_{L_{0}}\right)$ with energy $E_{L_{0}}$. Thus we will obtain

$$
H_{P}(q, \dot{q}, b)=\frac{1}{2} \eta^{\mu \nu}(q, \dot{q}) b_{\mu} b_{\nu}+E_{L_{0}}(q, \dot{q}) \text {. }
$$

Therefore, we have recovered the reduced dynamical system $\left(R, \omega_{R}, \Gamma_{R}\right)$ as a generalized symplectic reduction of a symplectic manifold (see Ref. 13 and references therein for more details on generalized reduction).

\section{Local expressions}

Because the two-form $\omega$ is presymplectic, it is possible to find local Darboux coordinates $\left(q^{i}, p_{i}, z^{\mu}\right)$ such that $\omega=d q^{i} \wedge d p_{i}$. The coordinates $z^{\mu}$ can be considered as local coordinates along the fibers of $K$ because the vector fields $\partial / \partial z^{\mu}$ form a local basis for $\Gamma(K)$. Therefore, we can write locally the formula $\omega_{K}=d z^{\mu} \wedge d b_{\mu}$ where, as before, $b_{\mu}$ are local linear coordinates along the fibers of $K^{*} \rightarrow M$. Then we have 


$$
\Omega=d q^{i} \wedge d p_{i}+d z^{\mu} \wedge d b_{\mu} .
$$

Computing now the Hamiltonian vector field $\Gamma_{H_{P}}$ given by $i_{\Gamma_{H_{P}}} \Omega=d H_{P}$ and $H_{P}$ given by Eq. (6), we obtain

$$
\begin{aligned}
\Gamma_{H_{P}}= & \left(\frac{\partial H}{\partial p_{i}}+\frac{1}{2} b_{\mu} b_{\nu} \frac{\partial \eta^{\mu \nu}}{\partial p_{i}}\right) \frac{\partial}{\partial q^{i}}-\left(\frac{\partial H}{\partial q^{i}}+\frac{1}{2} b_{\mu} b_{\nu} \frac{\partial \eta^{\mu \nu}}{\partial q^{i}}\right) \frac{\partial}{\partial p_{i}} \\
& +\eta^{\mu \nu} b_{\nu} \frac{\partial}{\partial z^{\mu}}-\left(\frac{\partial H}{\partial z^{\mu}}+\frac{1}{2} b_{\nu} b_{\delta} \frac{\partial \eta^{\nu \delta}}{\partial z^{\mu}}\right) \frac{\partial}{\partial b_{\mu}}
\end{aligned}
$$

Then, if $H$ is projectable along the distribution $K$, i.e., $\partial H / \partial z^{\mu}=0$, then $\Gamma_{H_{P}}$ is tangent to $M$, because

$$
\left.\Gamma_{H_{P}}\right|_{\left\{b_{\mu}=0\right\}}=\frac{\partial H}{\partial p_{i}} \frac{\partial}{\partial q^{i}}-\frac{\partial H}{\partial q^{i}} \frac{\partial}{\partial p_{i}}
$$

and also, $\left.\left(i_{\Gamma_{H_{P}}} \Omega\right)\right|_{M}=d H$.

Notice that the projectability of $H$ is equivalent to the nonexistence of constraints and the PCA stabilizes at the first step because $M_{1}=M$. In fact, it is evident that the constraint submanifold $M_{1}$ defined in Eq. (3) is characterized as those points in $M$ such that $\partial H / \partial z^{\mu}=0$.

Remark: The local coordinates $z^{\mu}$ play the role of a local gauge fixing. In fact the equations $z^{\mu}=0$ define a local slice for the projection map $p: M \rightarrow R$. If $\left(q^{i}, p_{i}, z^{\mu}\right)$ is a coordinate system in a neighborhood $U$ of $m \in M$, and $p(m) \in R$, we have the local section $\sigma(V)=\left\{z^{\mu}=0\right\}$, where $V$ is the projection of $U$ by $p$. We also notice that the Poisson bracket in $\left(R, \omega_{R}\right)$ is precisely the Dirac bracket defined from the Poisson bracket $\{. .,\}_{P}$ in $P$ and the set of (second class) constraints $b_{\mu}, z^{\mu}$.

\section{An example: Linear Lagrangian systems}

Let $L_{0}$ be a linear Lagrangian system on $T Q$, i.e.,

$$
L_{0}=A_{i}(q) v^{i}-V(q),
$$

where $A=A_{i}(q) d q^{i}$ defines a one-form on $Q$. We will assume for simplicity that $F=d A$ is nondegenerate, that is, $\operatorname{det} F_{i j} \neq 0, F=F_{i j} d q^{i} \wedge d q^{j}$. It is clear that the Legendre transformation is given by $F_{L_{0}}(q, v)=(q, A(q))$, and then $F_{L_{0}}(T Q)=$ graph $A \subset T^{*} Q$ is a symplectic (or second class) submanifold of $T^{*} Q$. Denoting it by $M$, we must point out that the projection $\tau: T Q \rightarrow Q$ restricted to $M$ makes $\left(M,\left.\omega_{0}\right|_{M}\right)$ symplectically equivalent to $(Q, F)$, which is the space used in Ref. 14 to quantize the system.

It is clear that $\theta_{L_{0}}=\tau^{*} A$ and $\omega_{L_{0}}=\tau^{*} d A=\tau^{*} F$, where $\tau$ denotes the canonical projection $T Q \rightarrow Q$ and then $K=\operatorname{kcr} \omega_{L_{0}}=V(T Q)$ (the vertical distribution in $T Q$ ). Notice that the bundles $K^{*}$ and $T^{*} Q$ are isomorphic along the projection map $\tau$. Then, the total space $P$ can be identified with $P=T Q \oplus T^{*} Q$ with symplectic structure $\Omega$ defined by

$$
\Omega\left((u, v, \alpha),\left(u^{\prime}, v^{\prime}, \alpha^{\prime}\right)\right)=F\left(u, u^{\prime}\right)+\left\langle v, \alpha^{\prime}\right\rangle-\left\langle v^{\prime}, \alpha\right\rangle,
$$

where $(u, v, \alpha) \in T_{Q}\left(T Q \oplus T^{*} Q\right)=T Q \oplus T Q \oplus T^{*} Q$. In local coordinates $\left(q^{i}, v^{i}, p_{j}\right), \Omega$ is written as

$$
\Omega=F_{i j} d q^{i} \wedge d q^{j}+d v^{i} \wedge d p_{i}
$$



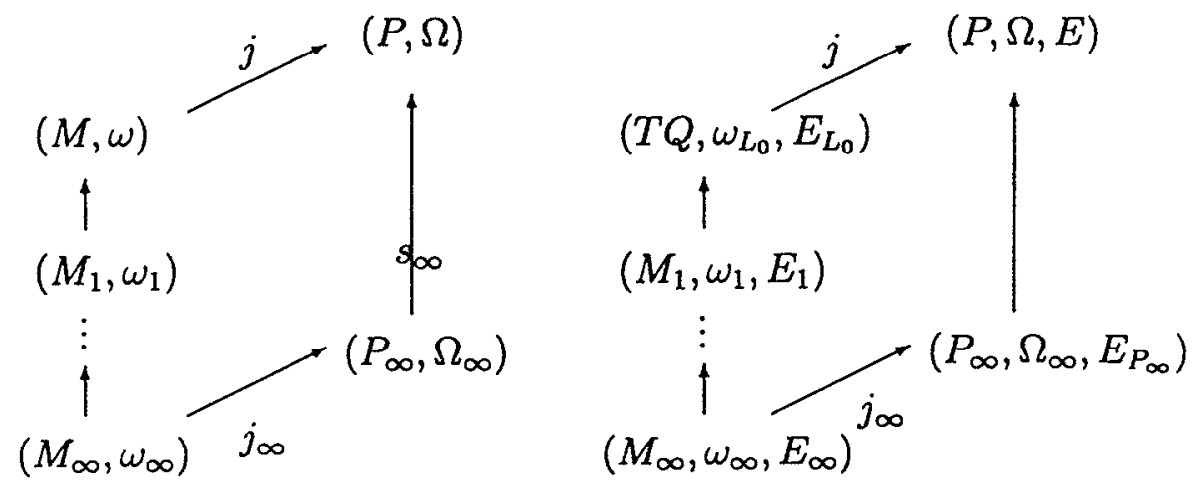

FIG. 1. Coisotropic regularization of higher order constrained presymplectic systems.

The energy will have the simple form $E_{L_{0}}=V$ and a regularized one is $H=\frac{1}{2} \eta^{i j}(q, v) p_{i} p_{j}$ $+V(q)$. Notice that the Poisson brackets defined by $\Omega$ are given by

$$
\left\{q^{i}, q^{j}\right\}=F^{i j} ; \quad\left\{q^{i}, v^{j}\right\}=\left\{q^{i}, p_{j}\right\}=0 ; \quad\left\{v^{i}, p_{j}\right\}=\delta_{i}^{j},
$$

where $F^{i j} F_{j k}=\delta_{k}^{i}$, and the equations of motion are simply

$$
\dot{q}^{i}=\frac{\partial V}{\partial q^{j}} F^{i j}+\frac{1}{2} F^{i j} \frac{\partial \eta^{k l}}{\partial q^{j}} p_{k} p_{l} ; \quad \dot{v}^{i}=\eta^{i j} p_{j} ; \quad \dot{p}_{i}=-\frac{1}{2} \frac{\partial \eta^{k l}}{\partial v^{i}} p_{k} p_{l} .
$$

Notice that restricted to $Q$, i.e., $p_{i}=v_{i}=0$, we obtain the usual dynamics defined by $L_{0}$, $\dot{q}^{i}=F^{i j} \partial V / \partial q^{j}$.

\section{E. Hamiltonian regularization of presymplectic systems with higher order constraints}

As was suggested in the previous sections, the ideas in Sec. II A-II C can be applied without major changes to presymplectic systems possessing higher order constraints. In such a case we can construct the coisotropic Hamiltonian regularization of the presymplectic system $\left(M_{\infty}, \omega_{\infty}, H_{\infty}\right)$ as in Sec. II A. We denote it by $\left(P_{\infty}, \Omega_{\infty}\right)$. It is well known that there exists a natural symplectic embedding $s_{\infty}:\left(P_{\infty}, \Omega_{\infty}\right) \rightarrow(P, \Omega),{ }^{15,16}$ where $(P, \Omega)$ denotes the coisotropic embedding regularization of $(M, \omega)$, making the diagram in Fig. 1 commutative. In other words, the local functions defining $P_{\infty}$ as a submanifold of $P$ are second class constraints with respect to the Poisson bracket $\{\ldots,\}_{P}$ and the local functions defining $M_{\infty}$ as a submanifold of $P_{\infty}$ are first class constraints. In addition, the regularized Hamiltonian system $\Gamma_{H_{P}}$ defined on $(P, \Omega)$ is such that the first class constraints $b_{\mu}$ defining the submanifold $M \subset P$, do not leave it invariant in general and the condition $\left.\Gamma_{P}\left(b_{\mu}\right)\right|_{\left\{b_{\mu}=0\right\}}=0$ is equivalent to the condition defining the primary constraint submanifold $M_{1} \subset M$. In fact, because of Eq. (6) we get

$$
\Gamma_{P}\left(b_{\mu}\right)=-\frac{\partial H}{\partial z^{\mu}}-\frac{1}{2} b_{\nu} b_{\delta} \frac{\partial \eta^{\nu \delta}}{\partial z^{\mu}}
$$

and on $b_{\mu}=0$, it implies $\partial H / \partial z^{\mu}=0$. Thus, the stability of the submanifold $M$ with respect to the regularized dynamical systems $\Gamma_{H_{P}}$ is equivalent to the first step in the PCA and we have proven that Hamiltonian regularization together with the generalized symmetries defined by the con- 
straints $b_{\mu}$ are equivalent to the original theory. Furthermore, we must notice that it is not always necessary to impose the generalized symmetry as shown, for instance, in example IV A.

If we apply this scheme to the presymplectic system $\left(T Q, \omega_{L_{0}}, E_{L_{0}}\right)$ defined by a singular Lagrangian $L_{0}$, we will obtain a coisotropic embedding $\left(T Q, \omega_{L_{0}}\right) \rightarrow(P, \Omega)$ and a sequence of constraint submanifolds $M_{k}$ defining eventually a final constraint submanifold $M_{\infty}$. The local functions defining $M_{\infty}$ as a submanifold of $P$ will be classified according to the discussion in the previous paragraph as first or second class constraints (in $P$ ).

It is remarkable that the constraint functions $b_{\mu}$ defining the coisotropic embedding $T Q \rightarrow P$ can become second class with respect to the final constraint submanifold $M_{\infty}$. For each direction on $M_{\infty}$ transversal to $K$ there is a pair $b_{\mu}, z^{\mu}$ of second class constraints. We must point out that we are just considering dynamical constraints in our analysis of the presymplectic system defined by $L_{0}$. Geometrical constraints arise from imposing the existence of solutions which are second order differential equations on the corresponding submanifolds (see Refs. 10, 11, and references therein for the discussion of singular Lagrangians with higher order constraints).

Finally, let us discuss how $\left(P_{\infty}, \Omega_{\infty}\right)$ can be considered as a reduced phase space of $(P, \Omega)$. Let $\nu\left(P_{\infty}\right)$ be the normal bundle to $P_{\infty}$ on $P$. It is clear that $\nu\left(P_{\infty}\right)$ is a symplectic bundle. Then, there is a symplectic diffeomorphism $\alpha: U \rightarrow P$, from a tubular neighborhood $U$ of the zero section of $P_{\infty}$ on $\nu\left(P_{\infty}\right)$ and a tubular neighborhood of $P_{\infty}$ on $P$ such that $\left.\alpha\right|_{P_{\infty}}=$ id. Let us choose a Lagrangian sub-bundle $L \subset \nu\left(P_{\infty}\right)$. Then, the previous diffeomorphism defines a coisotropic submanifold $C_{\infty}=\alpha(L \cap U) \subset P$ and a projection $\pi: C_{\infty} \rightarrow P_{\infty}$ such that ker $T \pi=\left.\operatorname{ker} \Omega\right|_{C_{\infty}}$. Hence, we obtain again that the second class submanifold $P_{\infty}$ is the generalized symplectic reduction of $P$ with respect to $C_{\infty}$. This method globalizes the local idea of splitting the second class constraints $\chi^{A}, A=1, \ldots, 2 r$, where $2 r=\operatorname{codim} P_{\infty}$, into two sets $\psi^{a}, a=1, \ldots, r$ and $\psi^{a+r}, a=1, \ldots, r$, each one defining coisotropic submanifolds whose intersection is $P_{\infty}$.

Finally, we should remark that the reduced space $R=M_{\infty} / K_{\infty}$ is obtained applying the mechanism of generalized symplectic reduction in two steps, first to the coisotropic submanifold $C_{\infty} \subset P$, and we obtain $P_{\infty}$, and secondly to the coisotropic submanifold $M_{\infty} \subset P_{\infty}$ obtaining $R$.

Remark: The mechanism of Hamiltonian regularization described here has no relation with the well-known method of converting second class constraints into first ones by duplicating them.

\section{COISOTROPIC LAGRANGIAN REGULARIZATION}

We will investigate now the existence of a Lagrangian structure on the Hamiltonian regularization $(P, \Omega)$ of the presymplectic system $\left(T Q, \omega_{L_{0}}, E_{L_{0}}\right)$. We will use the Hamiltonian function $H_{P}$ defined by Eq. (7). Notice that this discussion is independent of the existence or not of higher order constraints on the theory.

It is important to point out that $P$ will not be in general the tangent bundle of a manifold $M$. This is shown, for instance in example II $D$. Moreover, even if $P$ is a tangent bundle $P \cong T M$, a regular Lagrangian function $L_{\text {reg }}$ such that the closed two-form $\Omega$ will be the associated Cartan two-form $\omega_{L_{\mathrm{reg}}}$ may not exist. Finally, even if both conditions are satisfied, there is no a priori reason to think that $H_{P}$ will be the energy function of $L_{\text {reg }}$. Therefore, in general, a Lagrangian description for the regularized dynamical system $\left(P, \Omega, H_{P}\right)$ does not necessarily exist. If such a description exists, we will say that $L_{0}$ admits a (coisotropic) Lagrangian regularization.

We will show that if the (primary) distribution $K$ is the tangent distribution of a distribution $F$ such regularization exists. This situation corresponds to a particular case of type II Lagrangians, ${ }^{7,17}$ i.e., Lagrangians such that $S\left(\right.$ ker $\left.\omega_{L}\right)=V(T Q) \cap$ ker $\omega_{L}$. In this case $P$ is a tangent bundle and $L_{0}$ will admit (at least locally) a Lagrangian regularization. The distribution $F$ has to be an integrable distribution on $Q$ and we will assume that the quotient space $N=Q / F$ is a manifold and that the canonical projection $\rho: Q \rightarrow N$ is a submersion. The distribution $T F$ is the tangent distribution to $F$ on $T Q$ and is generated by vector fields of the form $X^{C}, Y^{V}$, where $X, Y$ are arbitrary vector fields in $F$ and $X^{C}, Y^{V}$ denote the complete and vertical liftings of $X$ and $Y$, 
respectively. It is well known that for any vector fields $X$ and $Y$ in $Q$, their complete and vertical liftings satisfy the commutation rules $\left[X^{C}, Y^{C}\right]=[X, Y]^{C},\left[X^{V}, Y^{C}\right]=[X, Y]^{V},\left[X^{V}, Y^{V}\right]=0$, and they preserve $S$. Because $S\left(X^{C}\right)=X^{V}$, it is clear that $S\left(\operatorname{ker} \omega_{L_{0}}\right)=V\left(\operatorname{ker} \omega_{L_{0}}\right)$, and therefore any Lagrangian function such that ker $\omega_{L_{0}}$ is a tangent distribution is necessarily a type II Lagrangian. Type II Lagrangians such that their kernels are tangent distributions are distinguished because only in this case (assuming the existence of a global dynamics) is it possible to obtain a Lagrangian description in the reduced space (see Ref. 7 for more details). If a Lagrangian function $L_{0}$ satisfies this property, it can be shown that $L_{0}$ induces a regular Lagrangian system in the quotient space $T Q / T F \cong T N$. For instance, the integrable almost tangent structure $S_{Q}$ in $T Q$ projects onto the integrable almost tangent structure $S_{N}$ in $T N$. Finally, it is important to remark that the Cartan one-form $\theta_{L_{0}}$ is projectable if and only if the Lagrangian function $L_{0}$ is projectable.

From the previous discussion it is natural to think that singular Lagrangian functions such that their kernel is a tangent distribution are the natural candidates to have a Lagrangian regularization. Therefore, we will assume in what follows that the kernel of the Cartan two-form is a tangent distribution $T F$ (this assumption can be relaxed as is shown in Example IV A).

\section{A. Local expressions}

It will be useful in what follows to have explicit expressions in local charts for some of the objects we are dealing with. In $Q$ we will consider local coordinates $q^{i}=\left(x^{a}, f^{\alpha}\right)$ adapted to $F, x^{a}$ being local coordinates in $N$ such that $\rho^{a}(q)=x^{a}$, and $f^{\alpha}$ are local coordinates along the leaves of $F$. Then we can define local coordinates in $T Q$ adapted to the integrable distribution $T F$ by $\left(q^{i}, \dot{q}^{i}\right)=\left(x^{a}, \dot{x}^{a} ; f^{\alpha}, \dot{f}^{\alpha}\right)$, where $\left(x^{a}, \dot{x}^{a}\right)$ are local coordinates in $T N$ and $\left(f^{\alpha}, \dot{f}^{\alpha}\right)$ are local coordinates along the fibers of $K=T F$. Because $K^{*}=(T F)^{*}$, we denote by $b_{\mu}=\left(g_{\alpha}, \dot{g}_{\alpha}\right)$ the system of local coordinates along the fibers of $K^{*}$ dual to the system defined by $\left(f^{\alpha}, \dot{f}^{\alpha}\right)$. Therefore, a local coordinate system in $P$ adapted to $K$ and $K^{*}$ is given by

$$
\left(x^{a}, \dot{x}^{a} ; f^{\alpha}, \dot{f}^{\alpha} ; g_{\alpha}, \dot{g}_{\alpha}\right)
$$

and in these coordinates, the symplectic two-form $\Omega$ given by Eq. (8) has the form

$$
\Omega=\omega_{L_{0}}+d f^{\alpha} \wedge d g_{\alpha}+d \dot{f}^{\alpha} \wedge d \dot{g}_{\alpha}
$$

The Hamiltonian $H_{P}$, Eq. (7), is written as

$$
H_{P}(x, \dot{x} ; f, \dot{f} ; g, \dot{g})=\frac{1}{2} \eta_{00}^{\alpha \beta} g_{\alpha} g_{\beta}+\frac{1}{2} \eta_{11}^{\alpha \beta} \dot{g}_{\alpha} \dot{g}_{\beta}+\eta_{01}^{\alpha \beta} g_{\alpha} \dot{g}_{\beta}+E_{L}(x, \dot{x}),
$$

where the metric $\eta$ on the bundle $K^{*}$ above can be chosen adapted to the distribution $T F$ as follows: let $\eta_{0}$ be a metric on the vertical bundle of $Q \rightarrow Q / F=N$, then $\eta_{00}^{\alpha \beta}=\eta_{11}^{\alpha \beta}=\eta_{0}^{\alpha \beta}(x)$, and $\eta_{01}=0$. From now on, we will omit the subindex 0 for this adapted metric.

\section{B. Tangent structure on the coisotropic embedding}

Let $M$ be a tubular neighborhood of the zero section of the vector bundle $F^{*} \rightarrow Q$. We will denote by $l: Q \hookrightarrow M$ the embedding of $Q$ on the zero section of $F^{*}$. In $M$ we can choose local coordinates $\left(q^{i}, g_{\alpha}\right)=\left(x^{a}, f^{\alpha}, g_{\alpha}\right)$. Let $T M$ be its tangent bundle, with local coordinates $\left(x^{a}, f^{\alpha}, g_{\alpha} ; \dot{x}^{a}, \dot{f}^{\alpha}, \dot{g}_{\alpha}\right)$. Then there is a local diffeomorphism $\Phi: T M \rightarrow P$ defined by

$$
\Phi\left(x^{a}, f^{\alpha}, g_{\alpha} ; \dot{x}^{a}, \dot{f}^{\alpha}, \dot{g}_{\alpha}\right)=\left(x^{a}, \dot{x}^{a} ; f^{\alpha}, \eta^{\alpha \beta} g_{\beta} ; \eta_{\alpha \beta} \dot{f}^{\beta}, \dot{g}_{\alpha}\right) \text {. }
$$

Notice that $\Phi$ exchanges $\dot{f}^{\alpha}$ with $g_{\alpha}$ [compare the right hand side of Eq. (15) with expression (12)]. Of course, this definition of the diffeomorphism $\Phi: T M \rightarrow P$, though useful for concrete 
computations, is local. Therefore, we should provide a global construction of the diffeomorphism $\Phi$ that will allow us to identify the coisotropic manifold $P$ with the tangent manifold $T M$. We will prove first the following lemma:

Lemma 1: Let $X \hookrightarrow Y$ and $X \hookrightarrow Z$ be two embeddings of $X$ in $Y$ and $Z$, respectively, and $\phi: X \rightarrow X$ a diffeomorphism. If $A: T_{X} Y \rightarrow T_{X} Z$ is an isomorphism of vector bundles such that $\left.A\right|_{T X}=T \phi$, then there exists a neighborhood $U$ of $X$ in $Y$, a neighborhood $V$ of $X$ in $Z$, and $a$ diffeomorphism $\Phi: U \rightarrow V$ such that $\left.\Phi\right|_{X}=\phi$ and $\left.T \Phi\right|_{T_{X} Y}=A$.

Proof: Let us consider a supplementary sub-bundle of $T X$ in $T_{X} Y$, defined, for instance, by using a metric $g$, i.e., $T_{X} Y=T X \oplus T X^{\perp}$. Consider the geodesic spray $\eta_{t}^{q}$ defined by $g$. We define the exponential mapping

$$
\begin{aligned}
\exp ^{8}: T X^{\perp} & \rightarrow Y \\
v & \mapsto \eta_{1}^{g}(v),
\end{aligned}
$$

where $\eta_{i}^{q}(v)$ is the geodesic such that $\dot{\eta}_{\phi}^{g}(v)=v$. The exponential mapping exp $p^{g}$ induces a diffeomorphism from a tubular neighborhood $W$ of $X$ in $T X^{\perp}$ to a tubular neighborhood $U$ of $X$ in $Y$. The image $A\left(T X^{\perp}\right)$ of $T X^{\perp}$ in $T_{X} Z$ defines a decomposition $T_{X} Z=T X \oplus A\left(T X^{\perp}\right)$. Let $h$ be a metric adapted to such a decomposition, then we use the exponential mapping $\exp ^{h}$, which is a diffeomorphism from the tubular neighborhood $A(W)$ in $A\left(T X^{\perp}\right)$ to a tubular neighborhood $V$ of $X$ in $Z$, to define

$$
\Phi\left(\left(\exp ^{g}\right)^{-1}(v)\right)=\exp ^{h}(A v)
$$

for any $v \in U$. Clearly, $\Phi$ is a diffeomorphism such that $\left.T \Phi\right|_{T_{X} Y}=A$.

Let us now consider the submersion $p: Q \rightarrow N$. We can pull back the tangent bundle $\tau_{N}: T N \rightarrow N$ along $\rho$ and we will obtain the bundle $\rho^{*}(T N)=\left\{(q, v) \in Q \times T N \mid \rho(q)=\tau_{N}(v)\right\}$, i.e., in local coordinates, the points in $\rho^{*}(T N)$ have the form $\left(x^{a}, f^{\alpha}, \dot{x}^{a}\right)$. We will denote this bundle by $X$ in the discussion to follow. Then, there is a canonical projection $p: T Q \rightarrow X$ defined by $p(U)=\left(\tau_{Q}(U), T \rho(U)\right)$, or in local coordinates, $p\left(x^{a}, f^{\alpha}, \dot{x}^{a}, \dot{f}^{\alpha}\right)=\left(x^{a}, f^{\alpha}, \dot{x}^{a}\right)$. Then, $T Q$ is a vector bundle over $X$ with projection $p$. We can consider the embedding $i: X \hookrightarrow T Q$ defined by the zero section of this bundle, and we can finally compose it with the zero section of the bundle $T M \rightarrow T Q$. We have defined in this way an embedding $X \hookrightarrow T M$. On the other hand we have the coisotropic embedding $j: T Q \hookrightarrow P$. Composing it with $i$, we have another embedding $X \hookrightarrow P$. In order to apply the previous lemma we need a linear isomorphism $A: T_{X}(T M) \rightarrow T_{X} P$. Clearly we have the vector bundle over $X$

$$
T_{X}(T M) \cong T X \oplus F^{*} \oplus\left(F \oplus F^{*}\right)
$$

with local coordinates $(U, \alpha, b, \beta), U \in T X, b \in F, \alpha, \beta \in F^{*}$; where $\alpha$ represents a tangent vector in the direction of the bundle $F^{*}$ over $X$ (the $g$ direction), and $b, \beta$ represent tangent vectors in the tangent directions to $(\dot{f}, \dot{g})$. Similarly we have

$$
T_{X} P \cong T X \oplus F \oplus(T F)^{*} \cong T X \oplus F \oplus\left(F^{*} \oplus F^{*}\right)
$$

where the first factor $F$ represents the tangent direction to $\dot{f}$ in $T Q$ and $(T F)^{*}=K^{*}$. Therefore, we can define the linear isomorphism

$$
A(U, \alpha, b, \beta)=(U, b, \alpha, \beta)
$$


Furthermore it is clear that $\left.A\right|_{T X}=\mathrm{id}_{T X}$. Hence, we can apply the lemma above to the diffeomorphism $\phi=\mathrm{id}_{X}$ and the vector bundle isomorphism $A$ given by Eq. (16); then there exists a diffeomorphism $\Phi$ from a neighborhood $U$ of $X$ in $T M$ to a neighborhood $V$ of $X$ in $P$. Therefore, we have proven the following theorem:

Theorem 1: Let $L_{0}$ be a singular Lagrangian such that ker $\omega_{L_{0}}$ is a tangent distribution TF and $\rho: Q \rightarrow Q / F=N$ is the canonical projection. If $j:\left(T Q, \omega_{L_{0}}\right) \rightarrow(P, \Omega)$ is a coisotropic embedding, then there exists a neighborhood $M$ of $Q$ in $K^{*} \rightarrow Q$ such that $P$ is diffeomorphic to $T M$ along the submanifold $\rho^{*}(T N)$.

Remark: Notice that the diffeomorphism $\Phi$ does not leave invariant the submanifold $T Q$, i.e., $T Q$ is a submanifold both in $T M$ and $P$, but $\Phi^{-1}(T Q)$ does not coincide with the submanifold $T Q$ in $T M$ defined by the zero section of the bundle $T M \rightarrow T Q$. The constraints defining $T Q$ in $P$ are $g_{\alpha}$ and $\dot{g}_{\alpha}$ but then, the submanifold $\Phi^{-1}(T Q) \subset T M$ is defined by $\dot{f}^{\alpha}=0$ and $\dot{g}_{\alpha}=0$. Then, when dealing with a Lagrangian regularization of a singular Lagrangian, the original system is recovered in the submanifold defined by the constraints $\dot{f}^{\alpha}, \dot{g}_{\alpha}$ and not in the submanifold defined by $g_{\alpha}$ and $\dot{g}_{\alpha}$.

\section{Construction of the symplectic structure in $T M$}

Let us consider the pullback of the two-form $\Omega$ in $P$ to $T M$ along the diffeomorphism $\Phi$. We obtain in this way a symplectic two-form $\Omega_{\mathrm{reg}}=\Phi^{*} \Omega$ in $T M$. In the adapted local coordinates (12), $\Omega_{\text {reg }}$ has the form

$$
\Omega_{\mathrm{reg}}=\frac{\partial^{2} L_{0}}{\partial \dot{x}^{a} \partial \dot{x}^{b}} d x^{a} \wedge d \dot{x}^{b}+\eta_{\alpha \beta} d f^{\alpha} \wedge d \dot{f}^{\alpha}+\eta^{\alpha \beta} d g_{\alpha} \wedge d \dot{g}_{\alpha} \text { thorizontal terms, }
$$

where the adapted metric $\eta$ in Sec. III A has been used for the construction. The horizontal part in the previous equation means that the contraction with any vertical vector on $T M \rightarrow M$ vanishes. Notice that the vertical distribution $V(T M)$ is a Lagrangian distribution for $\Omega_{\text {reg }}$, thus it is a good candidate for a Cartan two-form. .

The tangent structure in $T M$ is, in these adapted local coordinates

$$
S_{M}=\frac{\partial}{\partial \dot{x}^{a}} \otimes d x^{a}+\frac{\partial}{\partial \dot{f}^{\alpha}} \otimes d f^{\alpha}+\frac{\partial}{\partial \dot{g}_{\alpha}} \otimes d g_{\alpha} .
$$

Now, the question is when the symplectic two-form $\Omega_{\text {reg }}$ is the Cartan two-form of a Lagrangian function $L_{\text {reg }}$ in $T M$. In order to answer this question, we will make use of the geometrical description of the inverse problem in Lagrangian mechanics. Given a vector field $\Gamma$ which is a second order differential equation (SODE) in $T M$, necessary and sufficient conditions for the vector field $\Gamma$ to be the vector field solution of the Euler-Lagrange equations associated to a (locally defined) Lagrangian function $L_{\text {reg }}$ are that it must exists a two-form $\omega$ satisfying (Refs. 18 and 19 , see also Ref. 20 for a thorough discussion on the inverse problem)

(i) $\omega$ is closed and nondegenerate,

(ii) $\mathscr{L}_{\Gamma} \omega=0$,

(iii) $\omega\left(V_{1}, V_{2}\right)=0$ for any couple of vertical vector fields $V_{1}$ and $V_{2}$.

In the particular case we are discussing, $\omega=\Omega_{\text {reg }}$ is by construction, closed and nondegenerate. If we consider the vertical distribution $V(T M)$ in $T M$ generated by the vector fields of the form $Z=X^{a} \partial / \partial \dot{x}^{a}+F^{\alpha} \partial / \partial \dot{f}^{\alpha}+G_{\alpha} \partial / \partial \dot{g}_{\alpha}$, it is clear from Eq. (17), that $\Omega_{\text {reg }}$ is zero acting on two vertical fields.

The vector field $\Gamma=\Phi_{*}^{-1} \Gamma_{H_{P}}$ defined by the pullback of $\Gamma_{H_{P}}$ is Hamiltonian with respect to $\Omega_{\text {reg }}$ because of $\Phi_{*}^{-1} \Gamma_{H_{P}}=\Gamma_{\Phi * H_{P}}$ and $E_{\text {reg }}=\Phi^{*} H_{P}$ is the corresponding energy. On the other hand, since $\mathscr{L}_{\Gamma_{H_{P}}} \Omega=0$, then $\mathscr{L}_{\mathrm{r}} \Omega_{\mathrm{reg}}=0$, because 


$$
\mathscr{B}_{\Gamma} \Omega_{\mathrm{reg}}=\mathscr{C}_{\Phi_{*}^{-1} \Gamma_{H_{P}}}\left(\Phi^{*} \Omega\right)=\Phi^{*}\left(\mathscr{L}_{\Gamma_{H_{P}}} \Omega\right)=0
$$

Now, if we compute the pullback under $\Phi: T M \rightarrow P$ of the Hamiltonian function $H_{P}$ defined by Eq. (7) or by Eq. (14) we obtain

$$
E_{\mathrm{reg}}=\Phi^{*} H_{P}(x, \dot{x} ; f, \dot{f} ; g, \dot{g})=\frac{1}{2} \eta_{\alpha \beta}(x) \dot{f}^{\alpha} \dot{f}^{\beta}+\frac{1}{2} \eta^{\alpha \beta}(x) \dot{g}_{\alpha} \dot{\xi}_{\beta}+E_{L_{0}}
$$

From the structure of $E_{\text {reg }}$ we can readily conclude that the vector field $\Gamma$, the solution of the dynamical equation $i_{\Gamma} \Omega_{\text {reg }}=d E_{\text {reg }}$, is a SODE, and $E_{\text {reg }}$ defines the energy of a regular local Lagrangian $L_{\text {reg }}$ with associated SODE, the image by $\Phi_{*}^{-1}$ of $\Gamma_{H_{P}}$. Therefore, $\Omega_{\text {reg }}$ satisfies the conditions for the existence of a regular local Lagrangian $L_{\text {reg }}$ such that $\Omega_{\text {reg }}$ is precisely the Cartan two-form associated with it. Hence the expression for the Langrangian $L_{\text {reg }}$ is

$$
L_{\mathrm{reg}}(x, \dot{x}, f, \dot{f}, g, \dot{g})=\frac{1}{2} \eta_{\alpha \beta}(x) \dot{f}^{\alpha} \dot{f}^{\beta}+\frac{1}{2} \eta^{\alpha \beta}(x) \dot{g}_{\alpha} \dot{g}_{\beta}+L_{0}
$$

\section{Lagrangian regularization and Dirac's theory of constraints}

Once we have a Lagrangian regularization of a singular Lagrangian $L_{0}$ as is described, for instance, in the previous section, we can define the Legendre transformation $F_{\text {reg }}: T M \rightarrow T^{*} M$ which is a local diffeomorphism. In fact

$$
F_{\mathrm{reg}}\left(x^{a}, f^{\alpha}, g_{\alpha} ; \dot{x}^{a}, \dot{f}^{\alpha}, \dot{g}_{\alpha}\right)=\left(x^{a}, f^{\alpha}, g_{\alpha} ; \frac{\partial L_{0}}{\partial x^{a}}, \eta_{\alpha \beta} \dot{f}^{\beta}, \eta^{\alpha \beta} \dot{g}_{\beta}\right)
$$

using the regular Lagrangian (19). The map $F_{\text {reg }}{ }^{\circ} \Phi^{-1}: P \rightarrow T^{*} M$ is a symplectic diffeomorphism. In fact, this identification between $P$ and $T^{*} M$ can be obtained directly using the characterization of symplectic manifolds with Lagrangian fibrations. ${ }^{21}$

We already notice that it could happen that the first class constraints $b_{\mu} \circ \Phi$ defining $T Q$ inside $T M$ are not symmetries of $E_{\text {reg }}$; in such a case we have shown, Eq. (11), that restricting to the subspace of $T Q$ where this first class constraints are symmetries, is equivalent to start the PCA at the first step, i.e., that they define the submanifold $M_{1}$. Then we will reproduce the PCA directly in the presymplectic system $\left(T Q, \omega_{L_{0}}, E_{0}\right)$ described in Sec. II E (see Fig. 2).

On the other hand we can pull back the constraints $b_{\mu}$ to $T^{*} M$ using the local diffeomorphism $F_{\text {reg. }}$. In this way we will obtain a family of first class functions $p_{\mu}=b_{\mu}^{\circ} \Phi^{\circ} F_{\text {reg }}^{-1}$. Again, these first class functions do not have to be symmetries of the regularized Hamiltonian $H_{\text {reg }}$. If we impose that

$$
\left.\left\{H_{\text {reg }}, p_{\mu}\right\}\right|_{\left\{p_{\mu}=0\right\}}=0
$$

then we are defining a submanifold of $T^{*} Q$. This submanifold coincides with the first constraint submanifold $C_{1}$ in the ordinary Dirac's algorithm, i.e., is the image of $T Q$ under the Legendre transformation defined by $L_{0}$.

Finally, we must remark that the first class/second class decomposition in both approaches, the regularized Lagrangian setting and Hamiltonian Dirac's theory of constraints, that correspond to the left hand side and right hand side, respectively, in Fig. 2, and that are characterized globally by the submanifolds $P_{\infty}, S_{\infty}$ and the respective symplectic embeddings $s_{\infty}$ and $v_{\infty}$, are not related, in the sense that the natural map $\Pi_{\infty}: P_{\infty} \rightarrow S_{\infty}$ is not symplectic (see Fig. 2 again); then second class constraints in the Hamiltonian formalism can transmute into first class constraints in the Lagrangian setting and vice versa. 


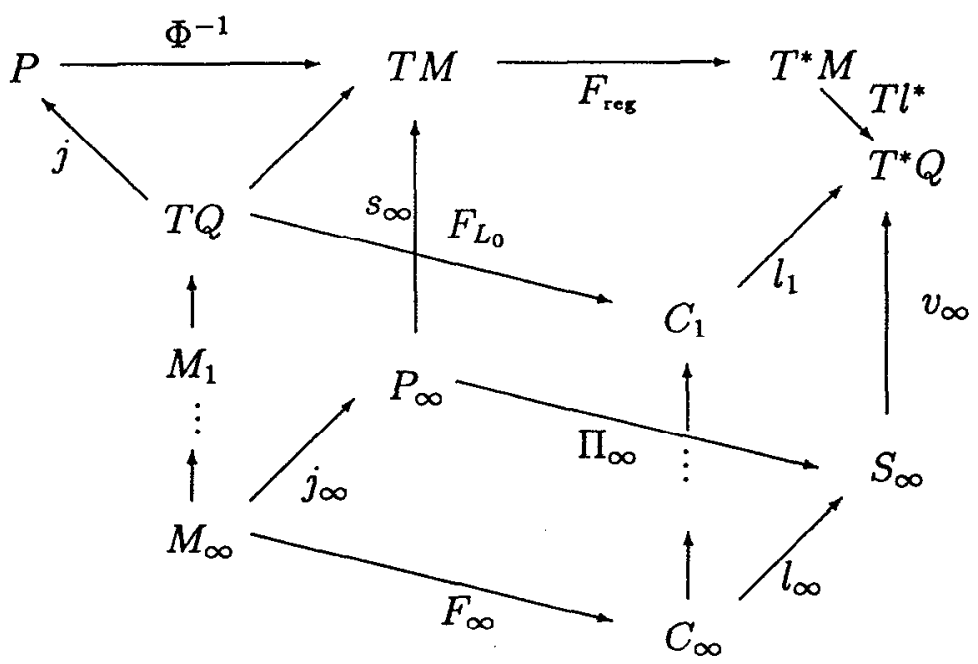

FIG. 2. Lagrangian regularization and Dirac's theory of constraints.

\section{EXAMPLES AND APPLICATIONS}

\section{A. A finite-dimensional example}

The finite-dimensional example proposed in the Introduction can be discussed, without restricting its generality, in two dimensions. Then, once one of the masses have been taken to be zero, we fix the other constants to 1 , and the Lagrangian $L_{0}$ can be written in the configuration space $\mathbb{R}^{2}$ with coordinates $(q, x)$ as

$$
L_{0}=\frac{1}{2}(\dot{q}+A(q, x))^{2}-V(q)
$$

and $\partial A / \partial x \neq 0$. This Lagrangian shares some of the geometrical features of the (infinitedimensional) Lagrangian of a pure Yang-Mills theory. After simple computations we get the Cartan one- and two-forms

$$
\theta_{L_{0}}=(\dot{q}+A) d q ; \quad \omega_{L_{0}}=d q \wedge d \dot{q}+\frac{\partial A}{\partial x} d q \wedge d x
$$

This Lagrangian is clearly degenerate and the kernel $K$ of the Cartan two-form $\omega_{L_{0}}$ is spanned by vector fields of the form

$$
Z=a \frac{\partial}{\partial x}-a \frac{\partial A}{\partial x} \frac{\partial}{\partial \dot{q}}+b \frac{\partial}{\partial \dot{x}}
$$

with $a, b$ arbitrary functions. The energy function of this system is given by

$$
E_{L_{0}}=\frac{1}{2} \dot{q}^{2}-\frac{1}{2} A(q, x)^{2}+V(q) .
$$

Notice that $V\left(\operatorname{ker} \omega_{L_{0}}\right)=\langle b \partial / \partial \dot{x}\rangle$ and then $S\left(\operatorname{ker} \omega_{L_{0}}\right)=V\left(\right.$ ker $\left.\omega_{L_{0}}\right)$. The Lagrangian $L_{0}$ is then of type II but $K$ is not a tangent algebra because of the term $a(\partial A / \partial x) \partial / \partial \dot{q}$. This is possible because there is no global dynamics for $L_{0}$. In fact, if we solve the dynamical equation (2) we 
obtain that a dynamical vector field $\Gamma$ exists only on the submanifold $M_{1}$ of $T \mathbf{R}^{2}$ defined by the constraint $\phi=\dot{q}+A(q, x)=0$. On this submanifold the dynamical vector field has the form

$$
\Gamma=\dot{q} \frac{\partial}{\partial q}+c \frac{\partial}{\partial x}+\left(A \frac{\partial A}{\partial q}-\frac{\partial V}{\partial q}-c \frac{\partial A}{\partial x}\right) \frac{\partial}{\partial \dot{q}}+d \frac{\partial}{\partial \dot{x}}
$$

again with $c, d$ arbitrary functions. In addition the restriction of $\omega_{L_{0}}$ to the submanifold $M_{1}$ vanishes identically, then the reduced space will have just one point. Thus the PCA applied to the presymplectic system $\left(T R^{2}, \omega_{L_{0}}, E_{L_{0}}\right)$ gives a trivial answer. The same happens if we apply the ordinary Dirac's theory of constraints in the Hamiltonian formalism. The image of the Legendre transformation gives the submanifold $C_{1}=\left\{p_{x}=0\right\}$ of $T^{*} \mathbb{R}^{2}$. The stability of the primary constraint $\phi=p_{x}$ gives the secondary constraint $\psi=p_{q}$ and $C_{2}=\left\{p_{x}=p_{q}=0\right\}$. The reduced phase space again reduces to a point. However, we must remark that $L_{0}$ is invariant under $K$, and $\theta_{L_{0}}$ and $S$ are projectable under $K$. The quotient space $R=T \mathbb{R}^{2} / K$ can be identified with $T \mathbb{R}$ (fixing, for instance, the coordinates $x, \dot{x}$ to zero), hence $L_{0}$ is reducible. The gauge algebra is an Abelian Lie algebroid and the quotient space can be readily computed integrating a basis of commuting vector fields in $K, \mathrm{Eq} .(22)$, and observing that points along the orbits can be parametrized by $x$ and $\dot{x}$. Thus, the quotient space inherits a tangent bundle structure and there is a well-defined Lagrangian on it. Denoting by $A_{0}(q)=A\left(q, x_{0}\right)$ we obtain the reduced Lagrangian function

$$
L_{R}=\frac{1}{\lambda}\left(\dot{q}+A_{0}\right)^{2}-V(q)
$$

the Cartan two-form is simply $\omega_{R}=d q \wedge d \dot{q}$, and the dynamical SODE is given by

$$
\Gamma_{R}=\dot{q} \frac{\partial}{\partial q}+\left(A_{0} \frac{\partial A_{0}}{\partial q}-\frac{\partial V}{\partial q}\right) \frac{\partial}{\partial \dot{q}}
$$

[compare with Eq. (24)]. It is remarkable that $E_{L_{0}}$ is not invariant under $K$ out of the submanifold $\phi=0$. We will choose as the true physical phase space the reduced space $T R$ with the reduced Lagrangian $L_{R}$. This is consistent with a physical interpretation of $x$ as an external control parameter for the magnetic field of the true system. On the other hand if we insist on the interpretation of this Lagrangian as a system with a negligible mass for the $x$ component, then we must restore this mass, or in other words, we have to regularize the system.

The coisotropic regularization of this system is obtained as follows. The vector bundle $K \rightarrow T \mathrm{R}^{2}$ is trivial, then $K \cong T \mathrm{R}^{2} \times \mathrm{R}^{2}$, hence, the dual bundle $K^{*} \rightarrow T \mathrm{R}^{2}$ is trivial and can be identified with $T \mathbf{R}^{2} \times \mathbf{R}^{2}$. We will denote the coordinates on $K^{*} \rightarrow T \mathbf{R}^{2}$ as $(q, x, \dot{q}, x ; \varphi, \psi)$. The symplectic two-form $\Omega$, Eq. (13), on the coisotropic embedding $T R^{2} \hookrightarrow T R^{2} \times R^{2}$ is given by

$$
\Omega=d q \wedge d \dot{q}+\frac{\partial A}{\partial x} d q \wedge d x+d x \wedge d \varphi+d \dot{x} \wedge d \psi
$$

The regularized Hamiltonian $H_{P}$, Eq. (14), can be chosen as

$$
H_{P}=\frac{1}{2} \dot{q}^{2}-\frac{1}{2} A^{2}+V(q)+\frac{1}{2} \varphi^{2}+\frac{1}{2} \psi^{2} .
$$

Even if the gauge algebra is not a tangent algebra, the diffeomorphism $\Phi$ constructed in Sec. III B exists and $K^{*}$ acquires a (nonunique) tangent bundle structure. In fact, defining $\Phi(\dot{x})=\varphi$ and $\Phi(\varphi)=\dot{x}$ we obtain that the total space of the bundle $K^{*}$ is diffeomorphic to $T \mathrm{R}^{3}$ with $\psi=\dot{\varphi}$. The Cartan two-form of the regularized system becomes

$$
\Omega_{\mathrm{reg}}=d q \wedge d \dot{q}+\frac{\partial A}{\partial x} d q \wedge d x+d x \wedge d \dot{x}+d \varphi \wedge d \dot{\varphi}
$$


and the regularized Lagrangian is given by

$$
L_{\mathrm{reg}}=\frac{1}{2}(\dot{q}+A(q, x))^{2}+\frac{1}{2} \dot{x}^{2}+\frac{1}{2} \dot{\varphi}^{2}-V(q) .
$$

The dynamical vector field for the regularized system is

$$
\Gamma=\dot{x} \frac{\partial}{\partial x}+\dot{q} \frac{\partial}{\partial q}+(\dot{q}+A) \frac{\partial A}{\partial x} \frac{\partial}{\partial \dot{x}}+\left(A \frac{\partial A}{\partial q}-\dot{x} \frac{\partial A}{\partial x}-\frac{\partial V}{\partial q}\right) \frac{\partial}{\partial \dot{q}}+\dot{\varphi} \frac{\partial}{\partial \dot{\varphi}}
$$

If we project on the subspace $x=x_{0}, \dot{\varphi}=0$, then $\dot{x}=0$ and the equations of motion reduce to Eqs. (26).

\section{B. Lagrangian regularization of the electromagnetic Lagrangian}

The configuration space $Q$ will be the Hilbert space $H^{1} \oplus \mathbf{H}^{1}$ where $H^{1}$ denotes the Sobolev space on $\mathbb{R}^{3}$ of class $(1,2)$ and $\mathbf{H}^{1}=H^{1} \oplus H^{1} \oplus H^{1}$. Points in $Q$ will be denoted by $A=\left(A_{1}, \mathbf{A}\right)$, $A_{\perp} \in H^{1}, \mathbf{A} \in \mathbf{H}^{1}$. The velocity phase space is $T Q=\left(H^{1} \oplus \mathbf{H}^{1}\right) \oplus\left(L^{2} \oplus \mathbf{L}^{2}\right)$, with $L^{2}$ the Hilbert space of all square integrable functions on $\mathbb{R}^{3}$. Points in $T Q$ will be denoted by $(A ; \dot{A})=\left(A_{\perp}, \mathbf{A} ; \dot{A}_{\perp}, \dot{\mathbf{A}}\right)$ with $\dot{A}_{\perp} \in L^{2}$ and $\dot{\mathbf{A}} \in \mathbf{L}^{2}$. The $3+1$ decomposed Maxwell Lagrangian for electromagnetism can be written as

$$
L_{0}(A ; \dot{A})=\frac{1}{2}\left\|\dot{\mathbf{A}}+\nabla A_{\perp}\right\|^{2}-\frac{1}{2}\|\nabla \wedge \mathbf{A}\|^{2},
$$

where $\|\mathbf{M}\|^{2}=\int|\mathbf{M}|^{2} d^{3} x$. More explicitly, $L_{0}$ can be written as

$$
L_{0}(A, \dot{A})=\frac{1}{2} \int\left(\dot{\mathbf{A}}^{2}+2 \dot{\mathbf{A}} \cdot \nabla A_{\perp}+\left|\nabla A_{\perp}\right|^{2}\right) d^{3} x-\frac{1}{2} \int|\nabla \wedge \mathbf{A}|^{2} d^{3} x
$$

A short computation give us the Cartan two-form $\omega_{L_{0}}$,

$$
\omega_{L_{0}}=\delta \mathbf{A} \wedge \delta \dot{\mathbf{A}}-\nabla \cdot \delta A_{\perp} \wedge \delta \mathbf{A}
$$

The energy $E_{L_{0}}$ is given by

$$
E_{L_{0}}(A, \dot{A})=\frac{1}{2}\|\dot{\mathbf{A}}\|^{2}-\frac{1}{2}\left\|\nabla A_{\perp}\right\|^{2}+\frac{1}{2}\|\nabla \wedge \mathbf{A}\|^{2}
$$

[compare the formulas (27), (28), and (29) with (20), (21), and (23)]. The dynamical condition $i_{\Gamma} \omega_{L_{0}}=d E_{L_{0}}$ is satisfied for some $\Gamma$ iff the following condition is satisfied:

$$
\boldsymbol{\nabla}\left(\dot{\mathbf{A}}+\nabla A_{\perp}\right)=0,
$$

i.e., there is a dynamical equation of motion only in the submanifold defined by the Gauss law. The primary constraint submanifold $M_{1}$ is given in the Lagrangian picture by the Gauss law, Eq. (30) (which becomes a primary constraint in this approach, contrary to what happens in the Hamiltonian picture, where it appears as the secondary constraint) and then it is easy to check that the dynamical vectors $\Gamma$ defined by the relations before leave invariant this constraint, then there are no further constraints.

Computing now the kernel of $\omega_{L_{0}}$, i.e., vector fields $Z$ such that $i_{Z} \omega_{L_{0}}=0$, we obtain

$$
Z=\alpha_{\perp} \frac{\delta}{\delta A_{\perp}}-\nabla \alpha_{\perp} \cdot \frac{\delta}{\delta \dot{\mathbf{A}}}+\beta_{\perp} \frac{\delta}{\delta \dot{A}_{\perp}}, \quad \alpha_{\perp}, \beta_{\perp} \in L^{2} .
$$


Clearly $K=\operatorname{ker} \omega_{L_{0}}=L^{2} \oplus L^{2}$, then $L_{0}$ is a Lagrangian of type II but its kernel is not a tangent algebra because of the term $\nabla \alpha_{\perp} \cdot \delta / \delta \dot{\mathbf{A}}$ (notice again the similarity with the previous example). Clearly, the tangent structure

$$
S=\frac{\delta}{\delta \dot{A}_{\perp}} \otimes \delta A_{\perp}+\frac{\delta}{\delta \dot{\mathbf{A}}} \otimes \delta \mathbf{A}
$$

is invariant under $K$, then $S$ projects to $T Q / K \cong T \mathbf{H}^{1}$. Furthermore, both $L_{0}$ and $\theta_{L_{0}}$ are invariant under $K$ as in the example before. The reduced Lagrangian in $T \mathbf{H}^{1}$ is

$$
L_{R}(\mathbf{A}, \dot{\mathbf{A}})=\frac{1}{2}\|\dot{\mathbf{A}}\|^{2}-\frac{1}{2}\|\nabla \wedge \mathbf{A}\|^{2},
$$

which is obviously nondegenerate with Cartan two-form $\omega_{R}=\delta \mathbf{\Lambda} \wedge \delta \dot{\mathbf{A}}$ and energy $E_{R}=\frac{1}{2}\|\dot{\mathbf{\Lambda}}\|^{2}$ $+\frac{1}{2}\|\nabla \wedge \mathbf{A}\|^{2}$. The reduced vector field $\Gamma_{R}$ defined by $i_{\Gamma_{R}} \omega_{R}=\delta E_{R}$ is given by

$$
\Gamma_{R}=\dot{\mathbf{A}} \frac{\delta}{\delta \mathbf{A}}+\nabla \wedge \nabla \wedge \mathbf{A} \frac{\delta}{\delta \dot{\mathbf{A}}}
$$

It is obvious that this Lagrangian is not the one that we find if we remove first the gauge invariance of the theory. That Lagrangian is defined on the tangent space of the moduli space of orbits of vector potentials under the action of the group of gauge transformations. But the constraint submanifold $M_{1}$ defines a submanifold $M_{1} \cap T \mathbf{H}^{1}$, namely, the space of pairs $(\mathbf{A}, \dot{\mathbf{A}})$ such that $\boldsymbol{\nabla} \dot{\mathbf{A}}=0$, and the restriction of $\omega_{R}$ to it has a kernel spanned precisely by the group of gauge transformations. Once this final reduction is performed we get the true physical space.

Because the bundle $K \rightarrow T Q$ is trivial, we can identify $K$ with $\left(L^{2} \oplus L^{2}\right) \times Q$ and also with $K^{*}$. The regularized Lagrangian will be defined on the extended space $T Q \oplus L^{2} \oplus L^{2}$. The symplectic form will be $\Omega=\omega_{L_{0}}+\delta A_{\perp} \wedge \delta \alpha_{\perp}+\delta \dot{A}_{\perp} \wedge \delta \beta_{\perp}, \alpha_{\perp}, \beta_{\perp}$ being the fields along the fibers of $K^{*}$. The total space admits a tangent bundle structure using the immersion $\Phi: T\left(Q \oplus H^{1}\right) \rightarrow T Q \oplus L^{2} \oplus L^{2}$ given by $\Phi\left(\dot{A}_{\perp}\right)=\alpha_{\perp}$ and $\Phi\left(\alpha_{\perp}\right)=\dot{A}_{\perp}$, and $\beta_{\perp}=\dot{\alpha}_{\perp}$. The Cartan two-form of the regularized system becomes

$$
\Omega=\delta \mathbf{A} \wedge \delta \dot{\mathbf{A}}-\nabla \cdot \delta A_{\perp} \wedge \delta \mathbf{A}+\delta A_{\perp} \wedge \delta \dot{A}_{\perp}+\delta \alpha_{\perp} \wedge \delta \dot{\alpha}_{\perp}
$$

and the regularized Lagrangian becomes

$$
L_{\mathrm{reg}}=\frac{1}{2}\left\|\dot{\mathbf{A}}-\nabla A_{\perp}\right\|^{2}+\frac{1}{2}\left\|\dot{A}_{\perp}\right\|^{2}+\frac{1}{2}\left\|\dot{\alpha}_{\perp}\right\|^{2}-\frac{1}{2}\|\nabla \wedge \mathbf{A}\|^{2} .
$$

\section{The electron-monopole system}

We will consider now the third example quoted in the Introduction. The singular Lagrangian describing an electron moving in the field created by a magnetic monopole is given by ${ }^{3}$

$$
L_{0}=\frac{1}{2} m \dot{x}_{i} \dot{x}^{i}+i n \operatorname{Tr} \sigma_{3} s^{\dagger} \dot{s},
$$

where $x_{i}, i=1,2,3$ are coordinates on $\mathbb{R}^{3}$ and $s$ denotes an arbitrary unitary unimodular $2 \times 2$ matrix, i.e., an element of the $\mathrm{SU}(2)$ group.

We obtain this Lagrangian in the following way. The configuration space of a charged particle moving in the presence of a magnetic monopole is $\mathbb{R}_{0}^{3}=\mathbb{R}^{3}-\{\mathbf{0}\}$ and the equations of motion are

$$
m \ddot{x}_{i}=\frac{n}{r^{3}} \epsilon_{i j k} x_{j} \dot{x}_{k}
$$


where $r>0$ denotes the radial coordinate and $n$ is an integer such that $4 \pi n=e g$, $e$ being the electric charge and $g$ the magnetic charge of the monopole. It is well known that there is no Lagrangian function describing such a system on $T\left(\mathbb{R}_{0}^{3}\right)$, but there is a principal U(1)-bundle $Q$ over $\mathbb{R}_{0}^{3}$ such that there is a global Lagrangian $L_{0}$ on $T Q$ describing this system. The principal bundle $Q$ is given by $\mathbb{R}^{+} \times \mathrm{SU}(2)$ and the projection map $\pi: Q \rightarrow \mathbb{R}_{0}^{3}$ is given by $\pi(r, s)=\mathbf{x}$, where $\mathbf{x}=r \hat{\mathbf{x}}$ and $s \sigma_{3} s^{\dagger}=\sigma_{i} \hat{x}_{i}$. Notice that this is nothing but the Hopf fibration $S^{3} \rightarrow S^{2}$ times the identity on the radial coordinate $r$. The total space $Q$ is $\mathbb{C}^{2}-\{0\}$. The magnetic field created by the monopole defines a two-form $F_{0}$ on $\mathbb{R}_{0}^{3}$

$$
F_{0}=\epsilon_{i j k} \frac{x_{i} d x_{j} \wedge d x_{k}}{r^{3}}
$$

that can be pulled back to $Q, F=\pi^{*} F_{0}$. Because $H^{2}(Q)=0, F$ is exact and it is easy to see that $F=d A$ where $A=i \operatorname{Tr} \sigma_{3} \Theta$ where $\Theta=s^{\dagger} d s$ is the canonical left invariant Maurer-Cartan oneform on SU(2). In fact, $F$ can be written as

$$
F=i \operatorname{Tr} \sigma_{3} \Theta \wedge \Theta
$$

Then, in $T Q$ we can define the Lagrangian function Eq. (31) and then we compute the Cartan one-form $\theta_{L_{0}}$ to obtain

$$
\theta_{L_{0}}=(T \pi)^{*} \theta_{0}+n A
$$

where $T \pi: T Q \rightarrow T \mathrm{R}_{0}^{3}$ denotes the tangent map to the projection map $\pi$, and $\theta_{0}$ denotes the Cartan one-form on $T \mathbb{R}_{0}^{3}$ defined by the kinetic energy term $K_{0}=\frac{1}{2} m \dot{x}_{i} \dot{x}^{i}$. It is clear that

$$
\omega_{L_{0}}=(T \pi)^{*} \omega_{0}+n F,
$$

where $\omega_{0}=m d x^{i} \wedge d \dot{x}^{i}$ is the Cartan two-form of the kinetic energy term $K_{0}$. From the form of $\omega_{L_{0}}$ we see immediately that its kernel is given by the kernel of $(T \pi)^{*} \omega_{0}$, i.e., by the kernel of the tangent map to $T \pi$. This kernel is a tangent distribution as discussed in Sec. III and it is spanned by the vectors lying on the kernel of the map $T \pi$, this is $\operatorname{ker} \omega_{L_{0}}=T F$ and $F=\operatorname{ker} T \pi$. Notice that the bundle $F \rightarrow Q$ has rank 1 and it is spanned by the generator of the U(1) group acting on $S^{3}$. The bundle $F \rightarrow Q$ is trivial, as well as $T F$ and its dual $(T F)^{*}$. Then, the total space for the coisotropic embedding of $T Q$ is simply $T Q \times \mathbb{R}^{2}$, and the extended configuration space is simply $M=Q \times \mathbb{R}$, thus $T M=T Q \times T \mathbb{R}$. Denoting the coordinate along the new direction on $Q \times \mathbb{R}$ by $\psi$, we get that the regularized Cartan two-form has the expression

$$
\Omega_{\mathrm{reg}}=(T \pi)^{*} \omega_{0}+F+d \phi \wedge d \dot{\phi}+d \psi \wedge d \dot{\psi},
$$

where the coordinate $\phi$ is the angular coordinate on $Q$ corresponding to the action of the $U(1)$ defining the Hopf map. Notice that this two-form corresponds to a Lagrangian function $L_{\text {reg }}$ of the form

$$
L_{\mathrm{reg}}=L_{0}+\frac{1}{2} \dot{\phi}^{2}+\frac{1}{2} \dot{\psi}^{2}
$$

or equivalently, $L_{\mathrm{reg}}=K+i n \operatorname{Tr} \sigma_{3} s^{\dagger} \dot{s}$, where $K$ is a kinetic energy term on $Q \times \mathbb{R}$.

\section{ACKNOWLEDGMENTS}

The author A.I. wishes to acknowledge the partial financial support provided by CICYT under Project. No. PB92-0197 during the realization of this project and the hospitality of the Erwing Schrödinger Institute where the final stages of this work were completed. 
The authors also want to thank G. Marmo for helpful suggestions on the manuscript as well as the referee whose comments and remarks have helped to improve the final form of this article.

'A. Hanson, T. Regge, and C. Teitelboim, Constrained Hamiltonian systems (Accademia Nazionale dei Lincei, Roma, 1973), Vol. 22.

${ }^{2}$ R. Jackiw, Nucl. Phys. B, (Proc. Suppl.) A 18, 107-170 (1990).

${ }^{3}$ A. P. Balachandran, G. Marmo, B. S. Skagerstam, and A. Stern, Gauge Symmetries and Fiber Bundles. LNP 188, (Springer-Verlag, Berlin, 1983).

${ }^{4}$ L. A. Ibort and C. López-Lacasta, J. Phys. A 23, 4779-4792 (1990).

${ }^{5}$ P. A. M. Dirac. Lectures on Quantum Mechanics (Belfer Graduate School on Science, Yeshiva University, New York, 1964).

${ }^{6} \mathrm{M}$. Henneaux and C. Teitelboim, Quantization of Gauge systems (Princeton University, New York, 1992).

${ }^{7}$ F. Cantrijn, J. F. Cariñena, M. Crampin, and I. A. Ibort, J. Geom. Phys. 3, 353-400 (1986).

${ }^{8}$ M. Gotay and S. Sniatycki, Commun. Math. Phys. 82, 377-389 (1981).

${ }^{9}$ T. Courant, Trans. Am. Math. Soc. 319, 331-361 (1989).

${ }^{10}$ M. Gotay and J. M. Nester, Ann. Inst. Henri Poincaré A 30, 129-142 (1979); 32, 1-13 (1980).

"IJ. F. Cariñena, Fortschr. Phys. 38, 641-679 (1990).

${ }^{12}$ M. Gotay, Proc. Am. Math. Soc. 84, 111-114 (1982).

${ }^{13}$ G. Marmo, E. J. Saletan, A. Simoni, and B. Vitale, Dynamical Systems, a Differential Geometric Approach to Symmetry and Reduction (Wiley, New York, 1985).

${ }^{14}$ L. D. Faddeev and R. Jackiw, Phys. Rev. Lett. 60, 1692 (1988).

15 J. Sniatycki, Ann. Inst. Henri Poincaré A 20, 365-372 (1974).

${ }^{16} \mathrm{~J}$. Cariñena, J. Gomis, A. Ibort, and N. Román-Roy, J. Math. Phys. 26, 1961-1969 (1985).

${ }^{17}$ L. A. Ibort and J. Marin-Solano, J. Phys. A 25, 3353-3367 (1992).

${ }^{18}$ A. P. Balachandran, G. Marmo, B. S. Skagerstam, and A. Stern, Nucl. Phys. B 164, 427-444 (1980).

${ }^{19}$ M. Crampin, J. Phys. A 14, 2567-2575 (1981); M. Henneaux, Annals Phys. 140, 45-64 (1982).

${ }^{20}$ G. Morandi, C. Ferrario, G. Lo Vecchio, G. Marmo, and C. Rubano, Phys. Rep. 188, 1 (1988).

${ }^{21}$ G. Thompson, Lett. Math. Phys. 12, 241-248 (1986). 